\title{
Multiplicative isomorphisms at invertible matrices
}

\author{
A. Armandnejad and M. Jamshidi
}




\title{
MULTIPLICATIVE ISOMORPHISMS AT INVERTIBLE MATRICES
}

\author{
A. ARMANDNEJAD AND M. JAMSHIDI
}

Received 05 March, 2014

\begin{abstract}
Let $\mathbf{M}_{n}$ be the algebra of all $n \times n$ matrices with entries in the field of real numbers. For a matrix $Z \in \mathbf{M}_{n}$, it is said that a linear map $T: \mathbf{M}_{n} \rightarrow \mathbf{M}_{n}$ is multiplicative at $Z$ if $T(A B)=T(A) T(B)$ whenever $A B=Z$. In this paper we investigate some properties of multiplicative mapping at invertible matrices and also we characterize all multiplicative isomorphisms at invertible matrices. Then we give an example to show that multiplicativity at $I$ doesn't imply multiplicativity on $\mathbf{M}_{n}$, where $I \in \mathbf{M}_{n}$ is the identity matrix.
\end{abstract}

2010 Mathematics Subject Classification: 15A03; 15A04; 46L40

Keywords: multiplicative map, multiplicative isomorphisms at identity, multiplicative isomorphisms at invertible matrices

\section{INTRODUCTION}

Concerning linear maps on matrices, much work has been done on the problem described generally as follows. Let $W$ be a space of matrices, and $Q(A)$ be a quantity or property of matrices $A \in W$. Then determine the structure of linear maps $T$ : $W \rightarrow W$ which preserve $Q$ [i.e. $Q(T(A))=Q(A)$, for all $A \in W$ ]. Some aspects of this problem were surveyed in [4]. Moreover, when $R(A, B)$ is a relation between matrices $A, B \in W$, it is interesting to determine all linear maps $T: W \rightarrow W$ which preserve $R$ [i.e. $R(Q(A), Q(B))$ holds whenever $R(A, B)$ ]. For example, several authors have discussed preserving linear maps of majorization on some spaces of matrices (see [1], [2] and references therein). A linear map $T: \mathbf{M}_{n} \rightarrow \mathbf{M}_{n}$ is called multiplicative on $\mathbf{M}_{n}$ if $T(A B)=T(A) T(B)$, for all $A, B \in \mathbf{M}_{n}$. A well known fact about a multiplicative linear map on $\mathbf{M}_{n}$ is the following theorem which has a short and interesting proof in [6].

Theorem 1. Let $T: \boldsymbol{M}_{n} \rightarrow \boldsymbol{M}_{n}$ be a multiplicative bijective linear map. Then there exists an invertible matrix $S \in \boldsymbol{M}_{n}$ such that $T(A)=S A S^{-1}$, for every $A \in \boldsymbol{M}_{n}$.

Proof. (From [6], Theorem 1.1) Choose and fix a pair of nonzero vectors $u, y \in$ $\mathbb{R}^{n}$. Since $T$ is injective, we can find $z \in \mathbb{R}^{n}$ such that $T\left(u y^{t}\right) z \neq 0$. Define $S: \mathbb{R}^{n} \rightarrow$ $\mathbb{R}^{n}$ by $S x=T\left(x y^{t}\right) z, x \in \mathbb{R}^{n}$. The linearity of $S$ follows from the linearity of $T$. 
Moreover, $S$ is nonzero since $S u \neq 0$. For arbitrary $A \in \mathbf{M}_{n}$ and $x \in \mathbb{R}^{n}$ we have

$$
S A x=T\left((A x) y^{t}\right) z=T\left(A\left(x y^{t}\right)\right) z=T(A) T\left(x y^{t}\right) z=T(A) S x,
$$

and consequently,

$$
S A=T(A) S .
$$

Let $w$ be any vector in $\mathbb{R}^{n}$. Since $S u \neq 0$ and because $T$ is surjective we can find $B \in$ $\mathbf{M}_{n}$ such that $T(B) S u=w=S B u$. Thus, $S$ is surjective, and therefore invertible. It follows that $T(A)=S A S^{-1}, A \in \mathbf{M}_{n}$, as desired.

The concept of a linear multiplicative mapping at unity on $B(H)$ (where $B(H)$ is the algebra of all bounded linear operators on a Hilbert space $H$ ) was introduced in [3]. Also the authors characterized all linear multiplicative maps at unity which are weak-continuous and surjective on $B(H)$, where $H$ is an infinite dimensional Hilbert space. In fact, they showed that, such maps are automorphisms on $B(H)$. In [5] the authors proved the following theorem which we use it to prove the main results of this paper.

Theorem 2. Let $T: \boldsymbol{M}_{n} \rightarrow \boldsymbol{M}_{n}$ be a linear map. Then, $T$ preserves the set of all invertible matrices, if and only if there exist invertible matrices $A$ and $B$ such that,

(i) $T(X)=A X B, \forall X \in M_{n}$, or

(ii) $T(X)=A X^{t} B, \forall X \in \boldsymbol{M}_{n}$.

\section{MULTIPLICATIVE ISOMORPHISM AT INVERTIBLE MATRICES}

In this section we state some preliminaries about idempotent matrices to prove the main result of this paper. We recall that a matrix $P \in \mathbf{M}_{n}$ is idempotent if $P^{2}=P$. The following proposition shows that the set of idempotent matrices generates $\mathbf{M}_{n}$. The notation $E_{i, j}$ is used for the matrix which has 1 as $(i, j)$ entry and 0 elsewhere. It is easy to see that for every $i$ and $j(1 \leq i \neq j \leq n) E_{i, i}^{2}=E_{i, i}$ and $E_{i, j}^{2}=0$.

Proposition 1. There exists a basis for $\boldsymbol{M}_{n}$ which is contained in the set of idempotent matrices.

Proof. Assume $\left\{E_{i, j}: 1 \leq i, j \leq n\right\}$ is the standard basis of $\mathbf{M}_{n}$. Put $P_{i}=E_{i, i}$ and $P_{i, j}=E_{i, i}+E_{i, j}$ for every $i$ and $j(1 \leq j \neq i \leq n)$, then

$$
P_{i, j}^{2}=\left(E_{i, i}+E_{i, j}\right)^{2}=E_{i, i}^{2}+E_{i, i} E_{i, j}+E_{i, j} E_{i, i}+E_{i, j}^{2}=E_{i, i}+E_{i, j}=P_{i, j} .
$$

Therefore $P_{i}$ and $P_{i, j}$ are idempotent matrices. Put

$$
\mathscr{B}=\left\{P_{i}: 1 \leq i \leq n\right\} \cup\left\{P_{i, j}: 1 \leq j \neq i \leq n\right\} .
$$

It is easy to see that $\mathcal{B}$ is a basis for $\mathbf{M}_{n}$.

Definition 1. Let $Z \in \mathbf{M}_{n}$ be given. A linear map $T: \mathbf{M}_{n} \rightarrow \mathbf{M}_{n}$ is called multiplicative at $Z$ if $T(A B)=T(A) T(B)$ whenever $A B=Z$. 
Now, we provide some lemmas to obtain the main result.

Lemma 1. Let $T: \boldsymbol{M}_{n} \rightarrow \boldsymbol{M}_{n}$ be a linear map and let $Z \in \boldsymbol{M}_{n}$ be an invertible matrix. If $T$ is multiplicative at $Z$, then for every $A \in \boldsymbol{M}_{n}$, the following assertions are true:

(i) $T(Z) T(A)=T(Z A) T(I)$,

(ii) $T(A) T(Z)=T(I) T(A Z)$,

(iii) $T(Z) T\left(Z^{-1} A Z\right)=T(A Z) T(I)$,

(iv) $T\left(Z^{2}\right) T\left(Z^{-2} A\right)=T(A Z) T\left(Z^{-1}\right)$.

Proof. (i ) For every idempotent $P$,

$$
(Z+Z P)\left(I-\frac{1}{2} P\right)=Z=\left(Z-\frac{1}{2} Z P\right)(I+P) .
$$

Since $T$ is multiplicative at $Z$,

$$
\begin{aligned}
T(Z+Z P) T\left(I-\frac{1}{2} P\right) & =T\left(Z-\frac{1}{2} Z P\right) T(I+P) \Rightarrow \\
{[T(Z)+T(Z P)]\left[T(I)-T\left(\frac{1}{2} P\right)\right] } & =\left[T(Z)-T\left(\frac{1}{2} Z P\right)\right][T(I)+T(P)] \Rightarrow \\
T(Z) T(P) & =T(Z P) T(I) .
\end{aligned}
$$

Therefore by Proposition 1,

$$
T(Z) T(A)=T(Z A) T(I), \quad \forall A \in \mathbf{M}_{n} .
$$

(i i ) For every idempotent $P$,

$$
(I+P)\left(Z-\frac{1}{2} Z P\right)=Z=\left(I-\frac{1}{2} P\right)(Z+P Z) .
$$

An argument similar to that above implies $T(A) T(Z)=T(I) T(A Z)$.

(iii) In part ( $i$ ), put $Z^{-1} A Z$ instead of $A$.

(iv) For every idempotent $P$,

$$
\left(Z^{2}+Z^{2} P\right)\left(Z^{-1}-\frac{1}{2} P Z^{-1}\right)=Z=\left(Z^{2}-\frac{1}{2} Z^{2} P\right)\left(Z^{-1}+P Z^{-1}\right) .
$$

An argument similar to that above implies $T\left(Z^{2}\right) T\left(A Z^{-1}\right)=T\left(Z^{2} A\right) T\left(Z^{-1}\right)$. Putting $Z^{-2} A Z$ instead of $A$, then $T\left(Z^{2}\right) T\left(Z^{-2} A\right)=T(A Z) T\left(Z^{-1}\right)$.

Lemma 2. Let $T: M_{n} \rightarrow M_{n}$ be a bijective linear map that is multiplicative at an invertible matrix $Z$, then $T(I)=I$.

Proof. Since $T$ is multiplicative at $Z$,

$$
T(Z) T(I)=T(Z)=T(I) T(Z) .
$$

For every $A \in \mathbf{M}_{n}$, 


$$
\begin{aligned}
T(A) T(Z) T\left(Z^{-1}\right) & =T(I) T(A Z) T\left(Z^{-1}\right) & & \text { (by (ii), Lemma 1) } \\
& =T(I) T\left(Z^{2}\right) T\left(Z^{-2} A\right) & & \text { (by (iv), Lemma 1) } \\
& =T(Z) T(Z) T\left(Z^{-2} A\right) & & \text { (by }(i i), \text { Lemma 1) } \\
& =T(Z) T\left(Z^{-1} A\right) T(I) . & & \text { (by }(i), \text { Lemma 1) }
\end{aligned}
$$

And consequently,

$$
\begin{aligned}
T(I) T(A Z) & =T(A) T(Z) \\
& =T(A) T(Z) T(I)^{2} \\
& =T(A) T(Z) T\left(Z^{-1}\right) T(Z) \\
& =T(Z) T\left(Z^{-1} A\right) T(I) T(Z) \\
& =T(Z) T\left(Z^{-1} A\right) T(Z) \\
& =T(Z) T(I) T\left(Z^{-1} A Z\right) \\
& =T(Z) T\left(Z^{-1} A Z\right) \\
& =T(A Z) T(I)
\end{aligned}
$$

(by ( $i$ i ), Lemma 1)

(by $(v)$ )

(by ( $i$ i ), Lemma 1)

(by the above relation)

(by $(v)$ )

(by $(i i)$, Lemma 1$)$

(by (i i i), Lemma 1)

Now, since $T$ is surjective and $A \in \mathbf{M}_{n}$ is arbitrary, for every $B \in \mathbf{M}_{n}$, we have $B T(I)=T(I) B$. It implies that $T(I)=\lambda I$, for some nonzero $\lambda \in \mathbb{R}$. By $(i i)$ of Lemma $1, T\left(Z^{-1}\right) T(Z)=T(I)^{2}=\lambda^{2} I$ and hence $T(Z)$ is invertible. Now using (v) implies that $T(I)=I$.

The following theorems characterize the multiplicative isomorphism at invertible matrices.

Theorem 3. Let $T: \boldsymbol{M}_{n} \rightarrow \boldsymbol{M}_{n}$ be a bijective linear map and let $Z$ be an invertible non scalar matrix. Then $T$ is multiplicative at $Z$ if and only if $T$ is an automorphism i.e. there exists an invertible matrix $B \in M_{n}$ such that $T(X)=B X B^{-1}$ for all $X \in$ $M_{n}$.

Proof. As the sufficiency of the condition is easy to see, only we prove the necessity of the condition. By the proof of Lemma $1, T(Z)$ is invertible. For every invertible matrix $A \in \mathbf{M}_{n}, T(Z)=T\left(Z A^{-1} A\right)=T\left(Z A^{-1}\right) T(A)$ and hence $T(A)$ is invertible. Therefore $T$ preserves the set of invertible matrices and by Lemma 2, $T(I)=I$. Consequently, by Theorem 2, there exists an invertible matrix $B \in \mathbf{M}_{n}$ such that one of the following holds:

(i) $T(X)=B X B^{-1}, \forall X \in \mathbf{M}_{n}$, or (ii) $T(X)=B X^{t} B^{-1}, \forall X \in \mathbf{M}_{n}$.

If $n=1$ the statements $(i)$ and (ii) are the same. So we may assume that $n \geq 2$ and hence there exists an invertible matrix $A \in \mathbf{M}_{n}$ such that $A Z \neq Z A$. If the statement 
(i i ) holds, then

$$
\begin{aligned}
T(Z)=T\left(Z A^{-1} A\right) & =T\left(Z A^{-1}\right) T(A) \Rightarrow \\
B Z^{t} B^{-1} & =B\left(Z A^{-1}\right)^{t} B^{-1} B A^{t} B^{-1} \Rightarrow \\
A Z & =Z A .
\end{aligned}
$$

Which is a contradiction, so $T(X)=B X B^{-1}, X \in \mathbf{M}_{n}$, as desired.

Corollary 1. Let $T: M_{n} \rightarrow M_{n}$ be a bijective linear map and let $Z$ be an invertible non scalar matrix. Then $T$ is multiplicative at $Z$ if and only if $T$ is multiplicative on $M_{n}$.

Theorem 4. Let $T: M_{n} \rightarrow M_{n}$ be a bijective linear map and let $Z$ be an invertible scalar matrix. Then $T$ is multiplicative at $Z$ if and only if $T$ is an automorphism or anti automorphism i.e. there exists an invertible matrix $B \in \boldsymbol{M}_{n}$ such that $T(X)=$ $B X B^{-1}$ for all $X \in M_{n}$ or $T(X)=B X^{t} B^{-1}$ for all $X \in M_{n}$.

Proof. With an argument similar to the proof of Theorem 3, if $T$ is multiplicative at an invertible matrix $Z$, then $T$ is an automorphism or anti automorphism. It is easy to show that every anti automorphism is multiplicative at an invertible scalar matrix $Z$.

The following example shows that multiplicativity at $I$ doesn't imply multiplicativity on $\mathbf{M}_{n}$.

Example 1. Let $T: \mathbf{M}_{2} \rightarrow \mathbf{M}_{2}$ be the linear map defined as follows :

$$
T(X)=P X^{t} P^{-1}, \forall X \in \mathbf{M}_{2} \text {, where } P=\left(\begin{array}{ll}
0 & 1 \\
1 & 0
\end{array}\right) .
$$

Then $T$ is multiplicative at $I$ by Theorem 4. Consider the matrices:

$$
X=\left(\begin{array}{cc}
-1 & 0 \\
1 & 2
\end{array}\right) \text { and } Y=\left(\begin{array}{ll}
0 & 1 \\
0 & 0
\end{array}\right) \text {. }
$$

It is easy to see that $T(X Y) \neq T(X) T(Y)$. So $T$ is not multiplicative on $\mathbf{M}_{2}$.

\section{ACKNOWLEDGEMENT}

The authors are very grateful to the anonymous referee for his/her constructive comments.

\section{REFERENCES}

[1] A. Armandnejad, F. Akbarzadeh, and Z. Mohammadi, "Row and column majorization on $\mathbf{M}_{n, m}$," Linear Algebra and its Applications, vol. 437, pp. 1025-1032, 2012.

[2] A. Armandnejad and Z. Gashool, "Strong linear preservers of g-tridiagonal majorization on $\mathbb{R}^{n}$," Electronic Journal of Linear Algebra, vol. 23, pp. 115-121, 2012.

[3] J. P. Jia, W. S. He, and F. D. Zhang, "Multiplicative mapping at unit operator on $B(H)$," Scientia Magna, vol. 5, no. 4, pp. 57-64, 2009. 
[4] M. Marcus, "Linear transformations on matrices," J. Res. Nut. Bur. Standards, vol. 75, pp. 107-113, 1971.

[5] M. Marcus and R. Purves, "Linear transformations on algebra of matrices ii: The invariance of the elementary symmetric functions," Canad. J. Math, vol. 11, pp. 57-64, 1959.

[6] P. Semrl, "Maps on matrix spaces," Linear Algebra and its Applications, vol. 413, pp. 364-393, 2006.

Authors' addresses

A. Armandnejad

Vali-e-Asr University of Rafsanjan, Department of Mathematics, P. O. Box 7713936417, Rafsanjan, Iran

E-mail address: armandnejadevru.ac.ir

M. Jamshidi

Graduate University of Advanced Technology, P. O. Box 7631133131, Kerman, Iran

E-mail address: m.jamshidi@kgut.ac.ir 